\title{
Detecting the spandex injuries and therapies of stretched garments
}

\begin{abstract}
The purpose of this research is to observe the reason behind the damage of the elastic fibers of woven garments while manufacturing and to find out their remedies. Different types of elastic spandex woven garments were used in this research of different manufacturing stages. The reasons of the damages of the spandex woven garments were identified and their remedies were conversed. Spandex woven garments were damaged due to sewing and all other operations involved from cutting to finishing at factories. The content of the spandex fibers were identified using FTIR spectroscopy in accordance with the test method provided by AATCC 20A. Infrared radiation could show the IR spectral scan since IR radiation can pass through the fabric. This research was practiced based and the findings could be beneficial to the personnel involved in textile industries and for the manufacture of elastic woven garments.
\end{abstract}

Volume 5 Issue $3-2019$

\author{
Shariful Islam, Jarin Yasmin, Rowshanuzzaman \\ Kanon, AKM Saiful Islam, Faridul Islam \\ Department of Textile Engineering, Faculty of Science and \\ Engineering, City University, Dhaka, Bangladesh.
}

Keywords: Spandex damage, Elasticity, FTIR spectrum, IR radiation, Elasticity

\author{
Correspondence: Shariful Islam, Department of Textile \\ Engineering, Faculty of Science and Engineering, City University, \\ Dhaka, Bangladesh, Tel +880191 I 509933 , \\ Email Sharifultextiles@gmail.com
}

Received: December 30, 2018 | Published: June 13, 2019

\section{Introduction}

Different scholars worked related to these experiments at different times where literature review exposed different results. Some of which were similar and some of them were widely dissimilar. Due to the range of variables involved if one parameter was changed then the other property of the fabrics were changed. Scholar reported that elasticity of the spandex fibers can be obtained with proper temperature treatment. ${ }^{1}$ Heat setting closely compacted the spandex fibers inside it and gives a permanent structure. ${ }^{2}$ Researcher also reported that optimum strength can be obtained with proper temperature treatment. ${ }^{3}$ Temperature has a direct consequence on the strength properties of cotton spandex fabrics. ${ }^{4}$

Researcher also reported that the shrinkage properties of cotton spandex woven fabrics can be controlled with proper heat setting temperature. ${ }^{5}$ Heat application removed the gap between two successive chemical constituent and helps for thermal shrinkage. ${ }^{6}$ Researcher also reported that application of sustainable natural fibers can be ecofriendly. ${ }^{7}$ Sustainable natural fibers can be helpful to achieve warmth, comfort and all other welcoming properties on fabrics that can improve the overall performances of the fabrics. ${ }^{8}$ Researcher reported that elastic performances of the elastic garments can be enhanced with proper heat setting temperature. ${ }^{9}$ Researcher also experimented that elastic garments can be best suited for modern fashioning due to they can be fashioned as required shape of the body structure. ${ }^{10}$ Researcher experimented that spandex is composed of long chain polymers. ${ }^{11}$ These polymer ingredients can be compacted with proper heat setting temperature. ${ }^{12}$

Researcher also experimented that spandex fibers have long molecular chain. These long chain molecules have haphazard structure. ${ }^{13}$ They are composed of different polymer ingredients. ${ }^{14}$ Temperature application provides stability to the spandex yarns and provides a permanent amorphous structure from the randomness. ${ }^{15}$ Researcher also reported that strength has a direct relationship with the fiber content it have. If the polyester content percentage is increased the strength property is also increased..$^{16}$ Researcher also reported that cotton fibers can increase warmth, breathability, absorbency and good hand feel. On the other hand manmade polyester fibers can increase the property of abrasion resistance and strength. ${ }^{17}$ Researcher also reported that manmade fibers are made up of different polymer ingredients and these ingredients are made up of long chain polymer. ${ }^{18}$

\section{Material and methods}

\section{Materials used}

Cotton spandex woven fabrics of $98 \%$ cotton and $2 \%$ spandex were used in this research. Garments were collected from different stages as mentioned in Table 1. Figure 1 shows the spandex garments of different stages.

Table I Specifications of the cotton spandex woven garments of different stages

\begin{tabular}{llllll}
\hline S.N & Sample types & Composition & Construction & Weave & Weight (g/m2) \\
\hline I $\quad \begin{array}{l}\text { Elastic woven garments } \\
\text { of no spandex damage }\end{array}$ & $\begin{array}{l}98 \% \text { Cotton } 2 \% \\
\text { Spandex }\end{array}$ & $20 \times(10+40 \mathrm{D}) / 110 \times 65$ & $4 / 1$ Satin & 325 & 55 \\
2 & $\begin{array}{l}\text { Elastic woven garments } \\
\text { of heavy spandex damage }\end{array}$ & $\begin{array}{l}98 \% \text { Cotton } 2 \% \\
\text { Spandex }\end{array}$ & $20 \times(10+40 \mathrm{D}) / 110 \times 65$ & $4 / 1$ Satin & 325 \\
\end{tabular}




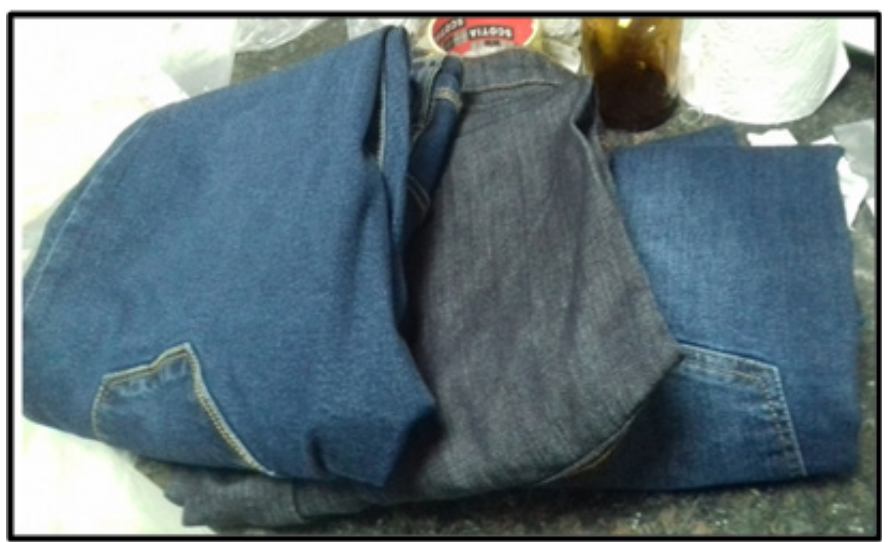

Figure I Cotton spandex woven garments of different stages.

\section{Method used}

AATCC 20A test method was used to assess the elastic performances of the specimen by the elastic content it had. IR radiation was exhibited an spectral scan using FTIR instrument in agreement with standard specified with AATCC $20 \mathrm{~A} .{ }^{19}$

\section{Instrument used}

Fiber content tester: Fiber content was tested with "TESTEX Fiber Content Analysis Tester TB300" in agreement with AATCC20 standard. Fibers were collected from fabric and placed at the instrument using glycerin to identify the fiber content. Figure 2 shows a fiber analysis tester. ${ }^{20}$

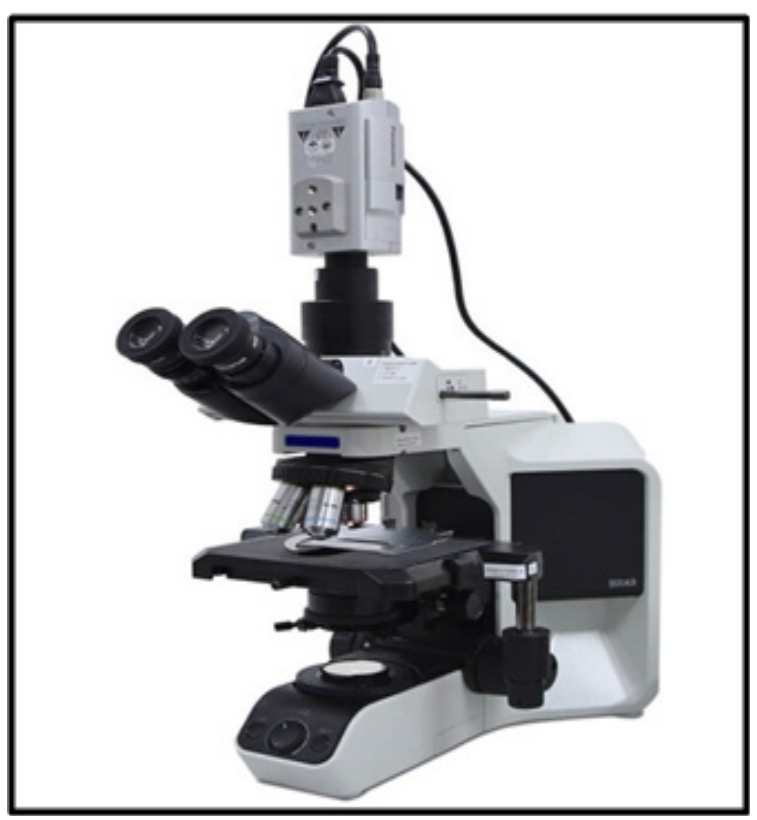

Figure 2 TESTEX Fiber Content Analysis Tester TB300, China, 2006.

FTIR instrument: FTIR instrument of "Nicolet IS 50 FTIR" was used in this research to assess the performances by its spectral scan. Figure 3 shows a FTIR instrument that was used in this research. ${ }^{21}$

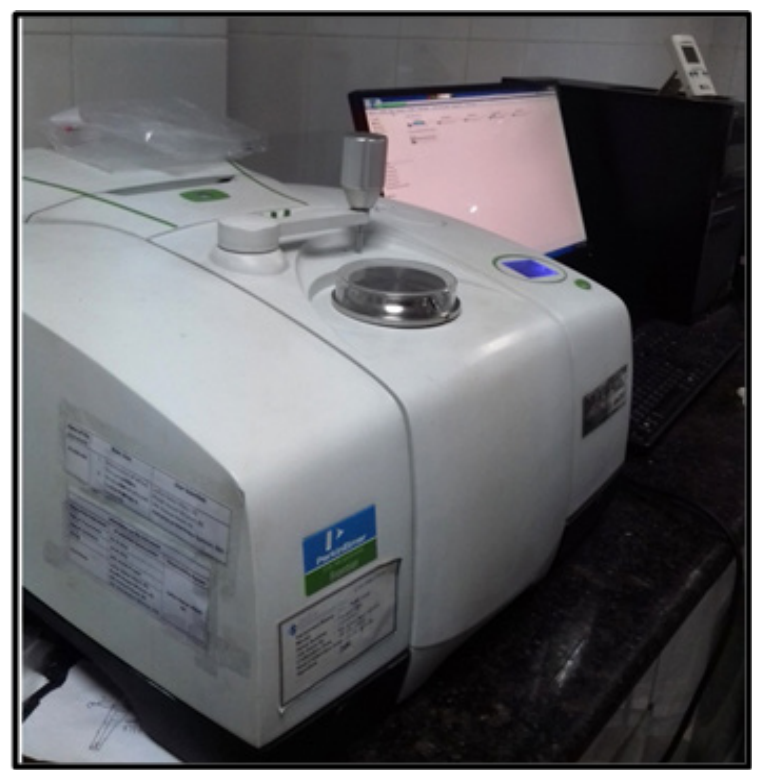

Figure 3 Nicolet IS 50 FTIR, USA, 2007.

\section{Causes of spandex injures}

Spandex damage means spandex missing from the fabrics. Therefore it gives an unwelcoming feel to the wearer. The regular wearer satisfaction is missed from the fabrics. Figure 4 shows the damages of spandex fibers in fabric. Figure 5 shows how spandex fibers were damaged due to sewing operation.

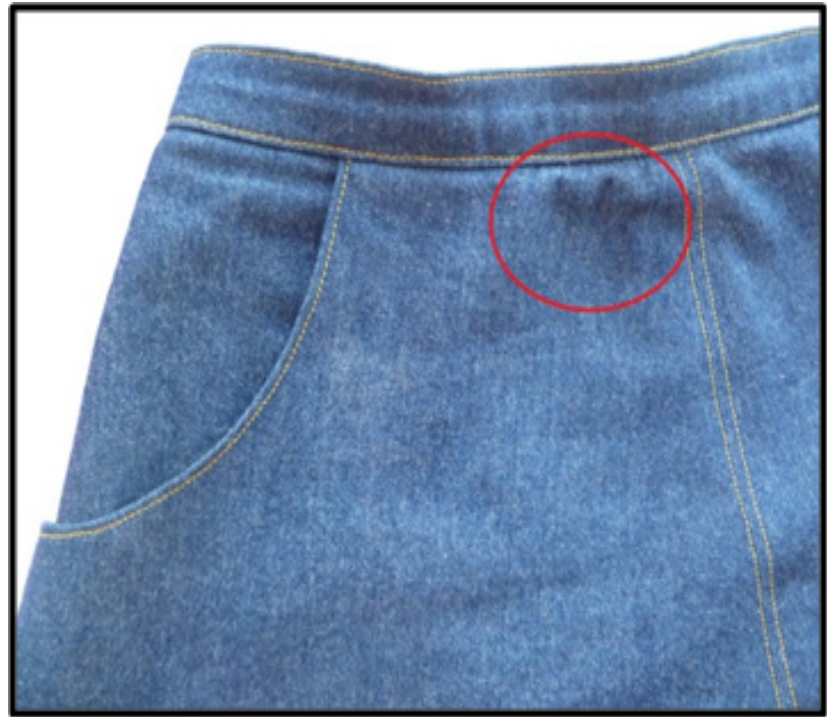

Figure 4 Spandex damage in the fabric.

Insufficient sewing allowance: Insufficient sewing allowance creates spandex damage in the fabric. Therefore, it is mandatory to give proper allowance while sewing.

Needle damage the spandex while sewing: Needle damages the spandex while sewing. Needle continuously strike the fabric can causes spandex damage.

More SPI more damage: More stitch per inch causes more spandex 
damage. The less the number of stitch in an inch the less the number of spandex breakage is.

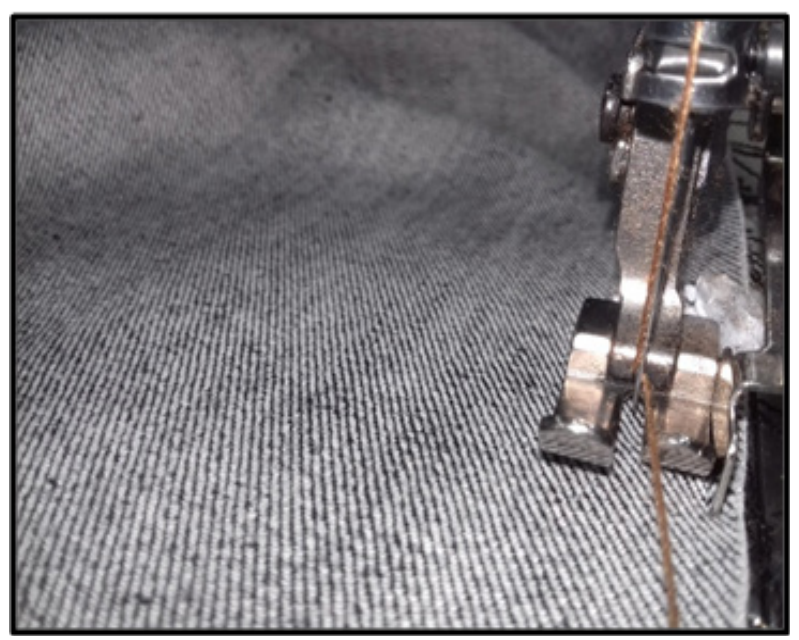

Figure 5 Spandex is damaged with the needle while sewing.

Too hot needle melt the spandex: While sewing needle becomes hot and this heated needle melt the spandex and creates damage.

Incorrect needle: Incorrect needle and incorrect machine mechanism damages more spandex in the garments.

Unnecessary stretch while sewing: Unnecessary stretching while sewing damages more spandex in the cloth.

More scrapping: More scrapping due to decoration purpose and destruction effect causes more spandex damage to the cloth.

Excess temperature wash: Excess temperature wash causes more spandex damage to the cloth.

Opened zipper: If the zipper remains while washing the cloth causes more spandex damage to the cloth.

Gas dryer: Application of gas dryer causes more spandex damage to the cloth since gas dryer has no temperature limit. On the other hand steam dryer has a constant temperature to $110^{\circ} \mathrm{C}$, so it causes less spandex damage to the cloth.

\section{Therapies of the spandex injures}

Less SPI: Less stitch per inch causes less spandex damage to the cloth. So, SPI can be minimized to reduce spandex damage to the cloth.

Less temperature wash: Less temperature wash can reduce spandex damage to the cloth. Higher temperature wash causes more thermal shrinkage therefore less temperature wash can reduce spandex damage.

Changing needle timely: Needle should be changed timely to reduce unwanted damage while sewing operation. Bent needle should also be changed while sewing.

Applying ball point needle: Application of golden ball point needle can reduce spandex damage to the cloth. These needles are specially prepared for sewing the elastic cloth.

Applying spandex protector: Spandex protector chemical can be used to reduce spandex damage to the cloth.
Applying rotary knife: Application of rotary knife reduces spandex damage since it does not directly force to the cloth rather it gives angular force which protects spandex from being damaged.

Never use hot gun: Never use hot gun to mend the elastic cloth. Hot gun damages more spandex so it should be avoided.

Avoid alkali, chlorine bleach and stone wash: Never use excess alkali to treat the spandex cloth as alkali is harmful to the cloth. Chlorine bleach is also destructive to the cloth so it should be avoided. Stone wash should also be avoided for the manufacturing of spandex cloth. Stone wash damages more spandex in the cloth.

Low temperature ironing: Use lower temperature while ironing the elastic cloth to protect the spandex from being damaged.

Apply dummy during scrapping: Dummy should be used while scrapping the garments with emery paper since it protects spandex from being damaged.

\section{The experiment}

FTIR experiment was conducted on cotton spandex woven garments. Infrared radiation was transmitted through the fabric to obtain the IR spectral scan. The elastic fiber content was identified using IR spectral scan. The FTIR instrument had a clamp that could be opened to place the garment for passing the IR radiation. Infrared radiation could pass through the fabric and IR radiation exhibited a spectral scan within its range. Figure 6 shows the technique of FTIR experiment of woven garments.

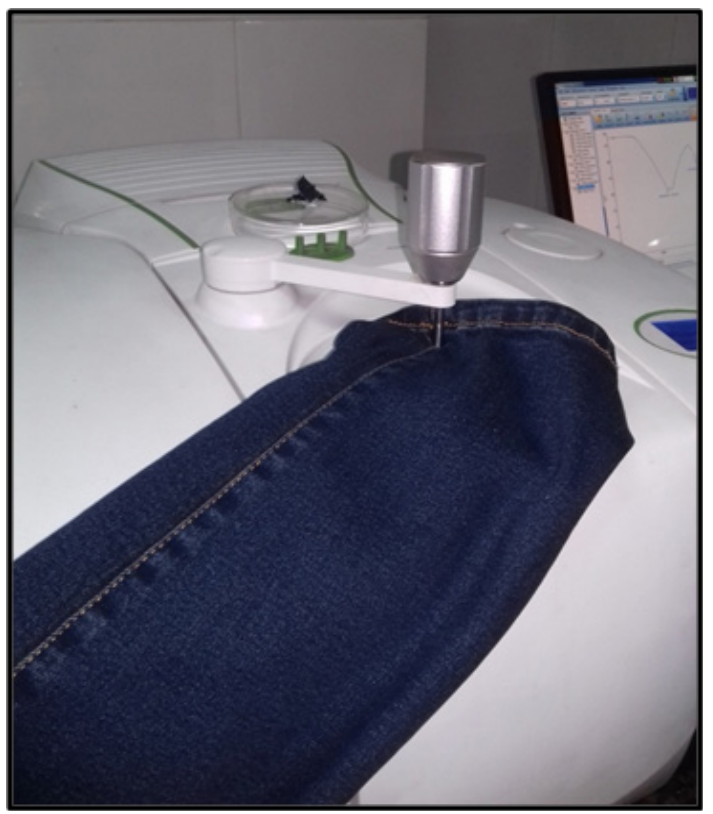

Figure 6 FTIR experiments of woven garments.

\section{Result and discussion}

IR radiations have a spectral range of 650 wave numbers to 4000 wave numbers. All the IR scans of all the samples were exhibited their spectral scan within this range. IR radiation could pass through the samples of different stages and exhibited different IR spectral proves. This radiation detected the elastic fiber content the sample has and also assessed the elastic performances. IR radiation could pass easily 
through the rejected sample since in this stage spandex fibers were missing so, infrared radiation could pass straightforwardly. On the other hand the sample with no spandex breakage has more spandex fibers so, IR radiation could not pass easily therefore it exhibited corkscrew IR scan. Figure 7 shows the IR spectral scan of the garments of no spandex damage. Figure 8 shows the IR spectral scan of the garments of severe spandex damage and Figure 9 shows the IR spectral scan of the garments of different stages.

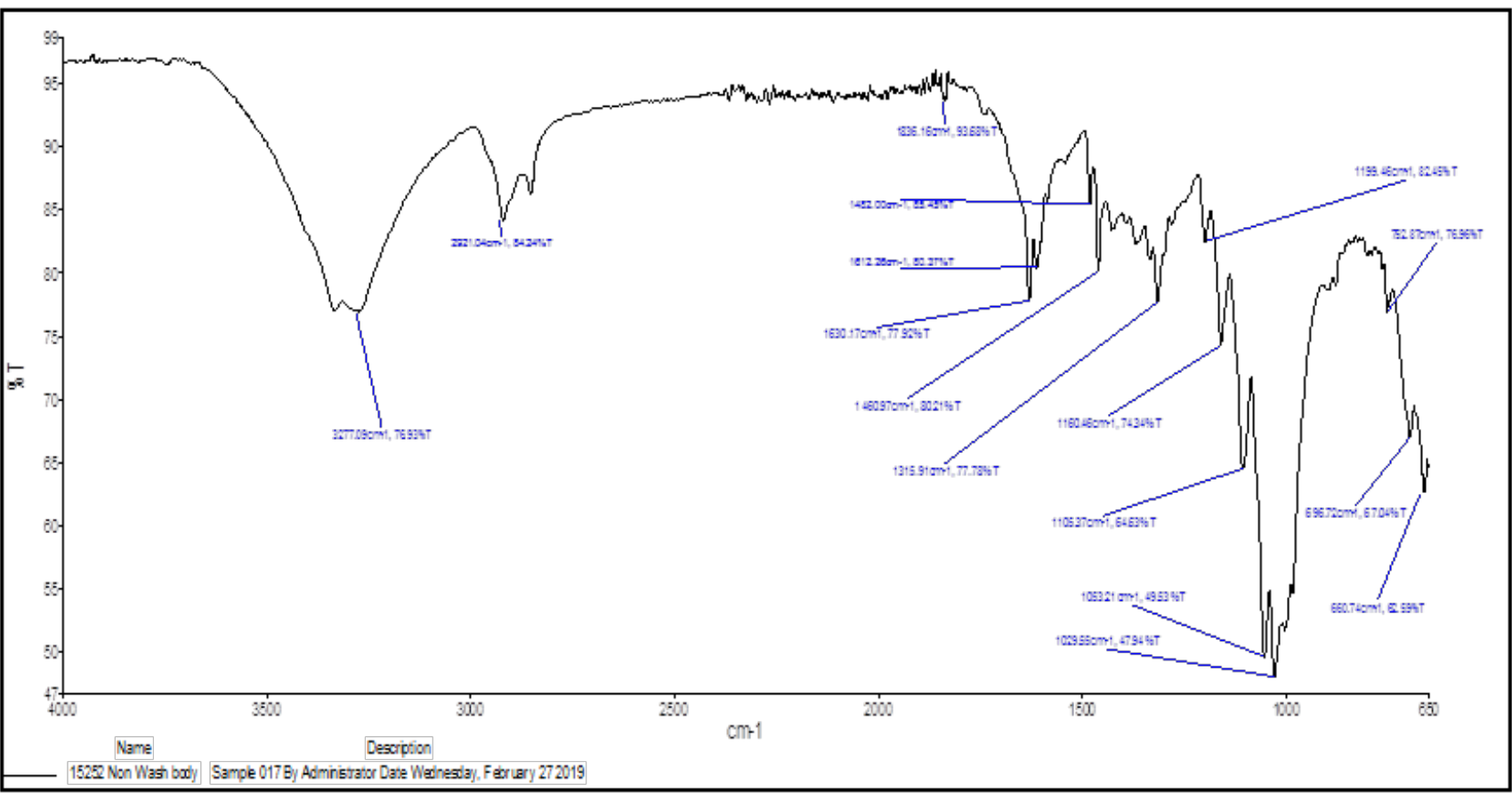

Figure 7 IR spectral scan of the garments of no spandex damage.

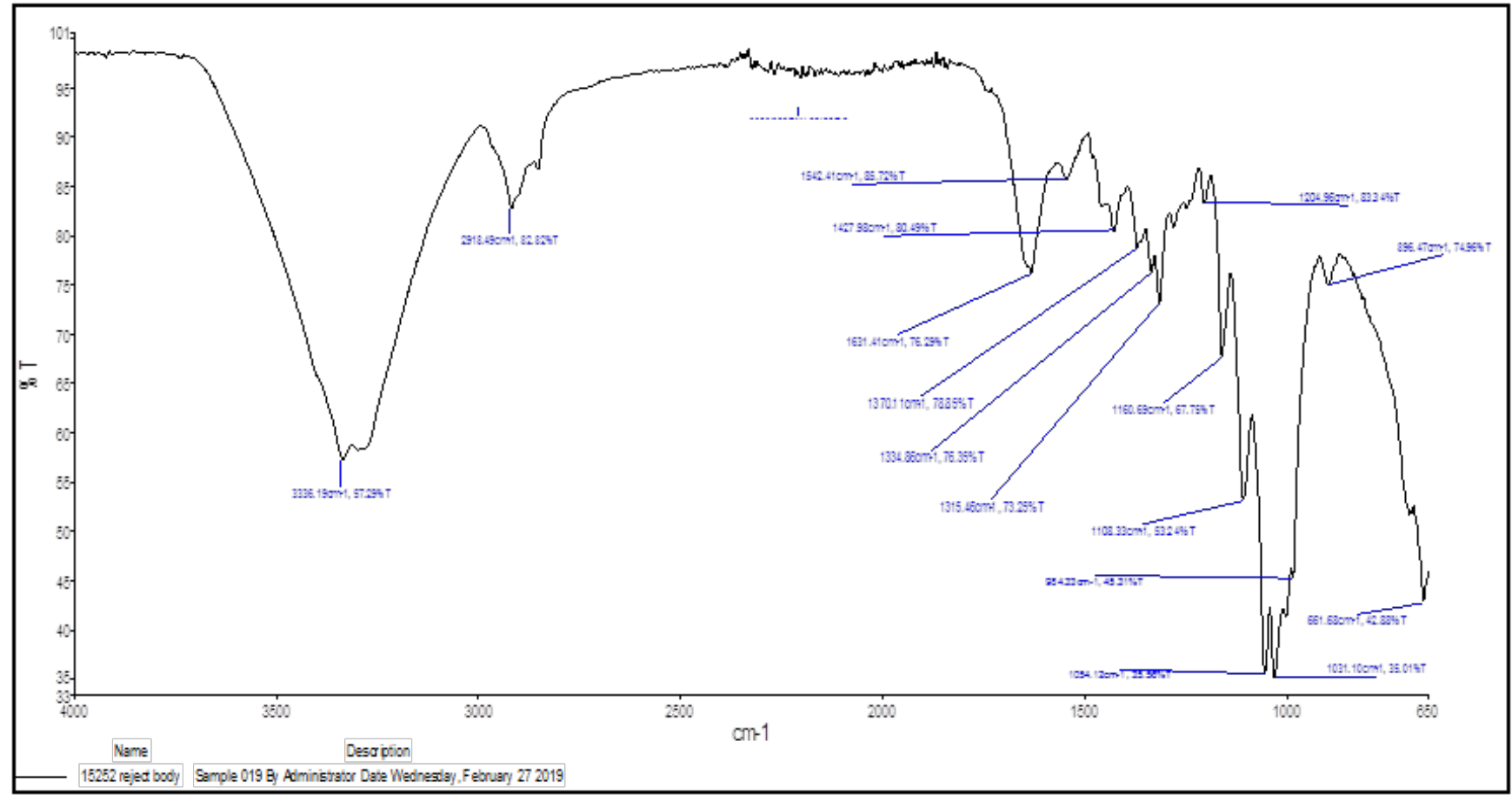

Figure 8 IR spectral scan of the garments of spandex damage. 


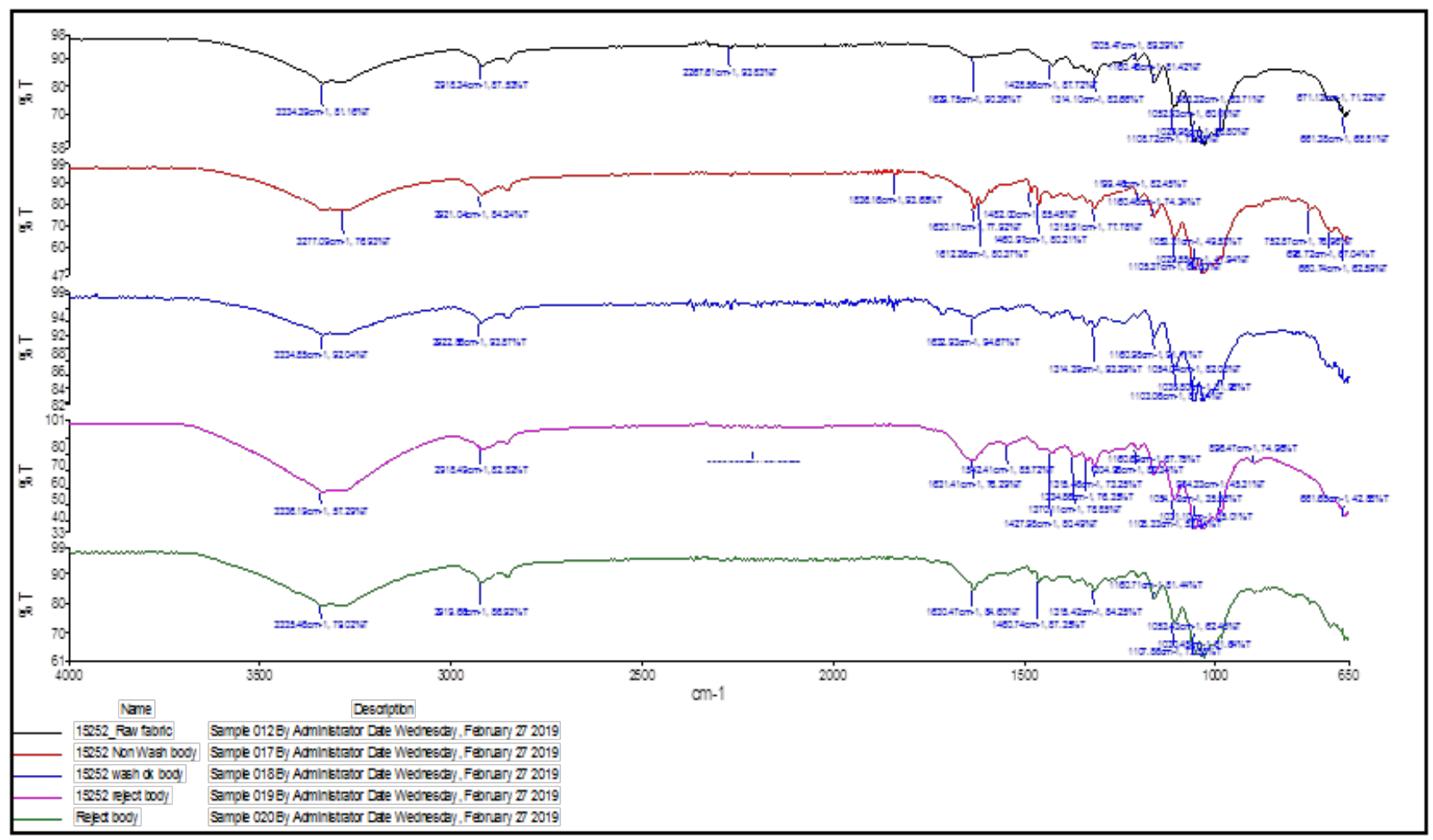

Figure 9 IR spectral scan of the garments of different stages.

\section{Conclusion}

It was understood from the research that the elastic fibers were very sensitive and their handlings were critical. The damages of the elastic fibers were happened at different manufacturing stages of the garments operation. Using FTIR instrument the elasticity of the spandex fibers were assessed through the transmission of the (IR) infrared radiation within its spectral scan region. IR radiation could pass easily through the samples of heavy spandex damage. Straightforward IR spectral scan was achieved due to the missing of the spandex fibers. Infrared radiation could not pass straightforwardly through the samples of no spandex damage rather they exhibited a corkscrew spectral scan. This research is practiced based and the findings are beneficial for the garments apprentices and professionals. This research opened possible ways for the scholar to further study in this field.

\section{Acknowledgments}

None.

\section{Conflicts of interest}

The authors declare that they have no competing interests.

\section{References}

1. Islam S, Alam SMM, Akter S. Identifying a suitable heat setting temperature to optimize the elastic performances of cotton spandex woven fabric. Research Journal of Textile and Apparel. 2018;22(3):260270.
2. Islam S. Attaining Optimum Strength of Cotton-Spandex Woven Fabric by Apposite Heat-Setting Temperature. Journal of The Institution of Engineers (India): Series C. 2018:1-6.

3. Islam S, Alam SMM, Akter S. The consequences of temperature on the shrinkage properties of cotton spandex woven fabrics. Journal of Textiles and Polymers. 2019;7(1):2-7.

4. Islam S, Mominul Alam SM. Investigation of the acoustic properties of needle punched nonwoven produced of blend with sustainable fibers. International Journal of Clothing Science and Technology. 2018;30(3):444-458.

5. Islam Shariful, Yasmin Jarin, Alam Syed, et al. Identifying the strength properties of cotton polyester blended woven fabrics of different fiber content. Research Journal of Material Sciences. 2019;7(2):1-6.

6. Besler N, Gloy YS, Gries T. Analysis of the heat setting process. IOP Conference Series: Materials Science and Engineering. 2016;141(1):012018.

7. Umar J, Hussain T, Ali Z, et al. Prediction modeling of compression properties of a knitted sportswear fabric using response surface method. World Academy of Science, Engineering and Technology, International Journal of Chemical, Molecular, Nuclear, Materials and Metallurgical Engineering. 2016;10(2):219-227.

8. Umar J,Hussain T, Maqsood M. Modeling the mechanical and compression properties of polyamide/elastane knitted fabrics used in compression sportswear. The Journal of The Textile Institute. 2016;107(10):1240 1252.

9. Baghdadi R, Alibi H, Fayala F, et al. Investigation on air permeability of 
finished stretch plain knitted fabrics. I. Predicting air permeability using artificial neural networks. Fibers and Polymers. 2016;17(12):2105-2115.

10. Pervez MN, Talukder ME, Datta MK, et al. The influence of annealing process on crystallinity and structural properties of cotton/spandex fabric. MATEC Web of Conferences. 2017;130:02001.

11. Chen Q, Ma P, Mao H, et al. The effect of knitting parameter and finishing on elastic property of PET/PBT warp knitted fabric. Autex Research Journal. 2017;17(4):350-360.

12. Zhang D, Chen W, Chen M. Research on ladder proof performance of melt spinning spandex blended knitted fabric. Advanced Textile Technology. 2017;(2)6.

13. Kant RITA, Chopra CHARU, Sisodia NIDHI. Need for ecofriendly finished textiles. International Journal of Applied Home Science. 2017;4(7):634-644.

14. Karthik T, Murugan R. Carbon footprint in denim manufacturing Sustainability in Denim. 2017:125-159.

15. Sabir T. Fibers used for high-performance apparel. High-Performance Apparel. 2018:7-32.
16. Islam Shariful, Ahmed Shaharia, Arifuzzaman Islam, et al. Relationship in between strength and polyester content percentage of cotton polyester blended woven fabrics. International Journal of Clothing Science. 2019;6(1):1-6.

17. Islam $\mathrm{S}$, Chowdhury $\mathrm{S}$, Akter $\mathrm{S}$. The experiential analysis of woven fabric for reproduction. Journal of Textile Science and Technology. 2018;4(1):18.

18. Cao H, Zulifqar A, Hua T, et al. Bi-stretch auxetic woven fabrics based on foldable geometry. Textile Research Journal. 2019;89(13):2694-2712.

19. AATCC Test Method 20A. Standard test methods for fiber content test of fabrics woven from stretch yarns. North Carolina, USA: AATCC International, Research Triangle Park; 2013:31-35.

20. Fiber Analyzing Instrument. "TESTEX Fiber Content Analysis Tester TB300", 2005. No.3, Daliantang Industrial Dt, Wan Jiang, Dongguan, 523000, P.R. China; 2014.

21. FTIR Instrument. "Nicolet IS 50 FTIR", 1015. Nicolet iS50 analytical FTIR spectrometer and software. Featuring purpose-built accessories and integrated software to easily solve analytical issues. USA. 